Recepción: 01 / 03/ 2018

Aceptación: 15 / 05 / 2018

Publicación: 07 / 08 / 2018

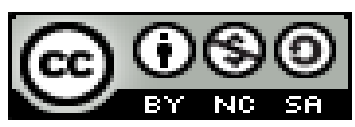

Ciencias de computación

Artículo Científico

\title{
Análisis de la tecnología WDS para diseño de redes Wi-Fi auto extensible en las arquitecturas hardware Atheros y Broadcom
}

Analysis of WDS technology for self-extending Wi-Fi network design in Atheros and Broadcom hardware architectures

Análise da tecnologia WDS para o design de redes Wi-Fi auto-extensíveis nas arquiteturas de hardware Atheros e Broadcom

Richar N. Muyulema Erazo I

rimuer1@gmail.com

Correspondencia: rimuer1@gmail.com

\footnotetext{
I Docente de la Escuela Superior Politécnica de Chimborazo, Facultad de Ciencias Pecuarias, Riobamba, Ecuador.
} 


\section{Resumen}

Este trabajo de investigación consiste en analizar la tecnología WDS (Wireless Distribution System) para diseño de redes Wi-Fi auto extensible en las arquitecturas hardware Atheros y Broadcom. Se utilizaron los Métodos: científico, deductivo y comparativo, para analizar el funcionamiento de WDS en Atheros y Broadcom, comparar los resultados obtenidos entre las dos arquitecturas en cuatro diferentes localidades en veinte (20) días de observaciones y determinar la arquitectura más óptima para la implementación de redes Wi-Fi auto extensible en el GAD de Cebadas. CommView for WiFi permitió capturar los paquetes de red, almacenarlos en una base de datos y establecer parámetros de comparación, con los datos obtenidos se procedió a calcular el Análisis de Varianza con Fisher a niveles de 0,01 y 0,05 de probabilidad y ratificar los resultados con InfoStat que permite procesar el ADEVA, y proporcionar la separación de medias y el nivel de significancia por la prueba de Tukey al nivel del $5 \%$ de probabilidad.

Palabras claves: Atheros, Broadcom, Hardware, WDS, Wi-Fi. 
Análisis de la tecnología WDS para diseño de redes Wi-Fi auto extensible en las arquitecturas hardware Atheros y Broadcom

\begin{abstract}
This research work consists in analyzing the WDS (Wireless Distribution System) technology for selfextending Wi-Fi network design in Atheros and Broadcom hardware architectures. Methods: scientific, deductive and comparative, to analyze the operation of WDS in Atheros and Broadcom, compare the results obtained between the two architectures in four different locations in twenty (20) days of observations and determine the most optimal architecture for the implementation of selfextending Wi-Fi networks in the GAD of Cebadas. CommView for WiFi allowed to capture the network packets, store them in a database and establish comparison parameters, with the obtained data we proceeded to calculate the Analysis of Variance with Fisher at levels of 0.01 and 0.05 probability and ratify the results with InfoStat that allows to process the ADEVA, and provide the separation of means and the level of significance by the Tukey test at the $5 \%$ probability level.
\end{abstract}

Key words: Atheros, Broadcom, Hardware, WDS, Wi-Fi 


\section{Introducción.}

La parroquia Cebadas no cuenta con servicios de comunicación inalámbrica en sus instituciones y organizaciones, por lo que es imperativo realizar estudios, análisis e implementación de nuevas tecnologías y arquitecturas existentes en el mercado actual de las comunicaciones, para cubrir necesidades como servicios de comunicación e internet en el Gobierno Autónomo Descentralizado (GAD) Parroquial. (PDOT. 2011). Como opción tecnológica y arquitectónica de comunicación en este punto geográfico se consideró la tecnología WDS (del inglés Wireless Distribution System), también conocido como modo repetidor, es un sistema que permite la interconexión inalámbrica entre los puntos de acceso AP (del inglés Access Point) de una red IEEE 802.11 (Román L. 2016), es decir, se utiliza para extender la cobertura de la red y utilizar direcciones MAC en lugar de asignaciones IP para conectar a los clientes (Zaggoulos, G. \& Nix, A. 2008.).

WDS permite la interconexión de los AP's de una red IEEE 802.11 sin necesidad de estar conectados a una red cableada, preservando la dirección MAC (equivalente a la dirección Ethernet cuando la trama llegue a la red cableada) de cada uno de los paquetes de los clientes a través de los distintos AP's. (Bandaancha. 2010). La conservación de la dirección MAC de los clientes provoca que el enlace WDS sea percibido por el resto de los equipos como un cable que no influye en la comunicación, lo que permite la interconexión de varios AP's que actuarían en modo repetidor de la señal entre ellos y también la comunicación con estaciones inalámbricas. (Rodríguez, R. 2010).

El WDS está integrado por nodos inalámbricos estáticos y sin limitaciones de almacenamiento o procesamiento. La función de estos nodos es encaminar los paquetes de datos provenientes de los usuarios móviles y hacerlos llegar al sistema cableado de distribución de Internet (Hiertz, G., 
Denteneer, D., Max, S., Taori, R., Cardona, J., Berlemann, L., Walke, B. 2010). Para realizar eficientemente esta función, el WDS debe ser conformado por dispositivos con capacidad suficiente para soportar grandes cantidades de tráfico. Normalmente, estos dispositivos utilizan las funciones definidas por el estándar IEEE 802.11n, o bien por el estándar IEEE 802.11ac, para alcanzar tasas de transmisión de hasta $1 \mathrm{~Gb} / \mathrm{s}$. Además, múltiples interfaces de red pueden ser implementadas en estos dispositivos para reducir interferencias e incrementar así la capacidad de la red. (Tung, L., Shih, W., Cho, T., Sun, Y., Chen, M. 2007.).

Esta investigación permitió analizar las arquitecturas hardware Atheros y Broadcom y determinar la más óptima para la implementación de una red Wi-Fi autoextensible con WDS, con esta tecnología, un punto de acceso puede funcionar como: punto de acceso, puente con otro punto de acceso, o ambas funciones. (Almeida, E. \& Coello, A. 2010). De esta manera fue posible crear una gran red inalámbrica dado que cada AP se conecta a cualquier otro AP disponible (que use WDS) y a cada AP se pueden conectar (de forma cableada o inalámbrica) la cantidad máxima que soporte el aparato (típicamente 256 equipos). (Tello, L. 2010).

\section{Materiales y métodos.}

\section{Localización de la investigación}

La presente investigación se desarrolló en las instalaciones del Gobierno Autónomo Descentralizado Parroquial de Cebadas, ubicado en la parroquia Cebadas, cantón Guamote, provincia de Chimborazo, país Ecuador. 


\section{Unidades experimentales}

Para el desarrollo de la presente investigación se utilizaron dos escenarios similares para las arquitecturas hardware Atheros y Broadcom en cuatro diferentes localidades llamadas: CEBADAS1, CEBADAS2, CEBADAS3 y CEBADAS4, durante veinte (20) días de observación.

Tabla 1. Grupos de IP definidos para la red propuesta

\begin{tabular}{|c|c|c|c|}
\hline Localidad & Dirección de Subred & Máscara de Subred & Default Gateway \\
\hline CEBADAS1 & 192.168 .1 .10 & 255.255 .255 .0 & 192.168 .1 .1 \\
\hline CEBADAS2 & 192.168 .1 .20 & 255.255 .255 .0 & 192.168 .1 .1 \\
\hline CEBADAS3 & 192.168 .1 .30 & 255.255 .255 .0 & 192.168 .1 .1 \\
\hline CEBADAS4 & 192.168 .1 .40 & 255.255 .255 .0 & 192.168 .1 .1 \\
\hline
\end{tabular}

\section{Procedimiento experimental}

En esta investigación se analizó la tecnología WDS para diseño de redes WI-FI auto extensible en las arquitecturas hardware (Redeweb, 2014) y Broadcom (Broadcom, 2015) y mediante un estudio del marco referencial se estableció tablas comparativas de las dos arquitecturas. Las características propias de cada arquitectura hace indispensable un estudio individual del rendimiento de cada una de ellas, factores como: la velocidad en la cantidad de paquetes transmitidos, paquetes recibidos, paquetes perdidos o error y Mbytes transferidos, fueron analizados para concluir verificando las fortalezas que ofrece cada arquitectura. Luego del análisis y escoger la arquitectura más óptima se establecieron parámetros para la implementación de redes Wi-Fi auto extensible en el GAD, para la presentación de los resultados de las dos arquitecturas se realizó el análisis generado por la red propuesta, (tabla 1). 
Para el procesamiento de la información y medir los paquetes recibidos $(\mathrm{Rx})$, transmitidos $(\mathrm{Tx})$, perdidos o error $(\mathrm{E})$ y Mbytes $(\mathrm{Mb})$ recibidos y transmitidos se procedió a montar un escenario similar para las dos arquitecturas y en cada una de ellas se hizo uso de los respectivos equipos, (tabla 2). (Flores, M. 2010).

Para la recolección de datos de las dos arquitecturas se investigó de tal manera que se verifico que todos los equipos móviles (Routers) que se usó para establecer la red Wi-Fi tengan configurada una dirección IP al conectarse, y que se encuentra en el mismo tipo de red, en este caso la red 192.168.1.xx. Para capturar los paquetes que transitan por la red se usó la herramienta CommView for WiFi. (Bellido, W. 2013).

Tabla 2. Equipos seleccionados para el diseño de la red Wi-Fi Auto extensible

\begin{tabular}{|c|c|c|}
\hline Arquitectura & Equipos & Protocolo / Estándar \\
\hline \multirow{3}{*}{ Broadcom } & $\begin{array}{lll}\text { Router } & \text { Wireless-G } & \text { Cisco-Linksys } \\
\text { WRT54GL } & & \end{array}$ & $\begin{array}{l}\text { IEEE 802.3, IEEE 802.3u, IEEE } \\
\text { 802.11g, IEEE 802.11b }\end{array}$ \\
\hline & Cable 3485 15Ft Wi-Fi M/M N & $802.11 \mathrm{a} / \mathrm{b} / \mathrm{g}$ Compatible \\
\hline & $\begin{array}{l}\text { Antena AIR802 } 2.4 \mathrm{GHz} \text { WiFi with } 7 \\
\text { Degrees Uptilt, Omni-directional 8dBi, } \\
\text { Mast Mount }\end{array}$ & $802.11 \mathrm{~b}, 802.11 \mathrm{~g}$ or 802.11 \\
\hline Atheros & $\begin{array}{l}\text { Router EnGenius EOC1650, Exterior, } 54 \\
\text { Mbps, } 2.4 \mathrm{GHz}, \text { PoE, } 200 \mathrm{~mW}, 24 \mathrm{dBm}\end{array}$ & $\begin{array}{l}\text { IEEE } 802.3 \text { (Ethernet) } \\
\text { IEEE } 802.3 \mathrm{u} \text { (Fast Ethernet) } \\
\text { IEEE } 802.11 \mathrm{~b} / \mathrm{g}(2.4 \mathrm{GHz} \text { WLAN }\end{array}$ \\
\hline
\end{tabular}

\section{Resultados y discusión.}

Paquetes Rx, arquitectura Atheros y Broadcom 
De los resultados obtenidos durante el periodo de monitoreo la la red Wi-Fi con la arquitectura Atheros se Rx 128129,45 paquetes, valor que difiere significativamente de la arquitectura Broadcom puesto que con ella se pudo Rx 35490,54 paquetes. (tabla 3).

En la localidad CEBADAS2 se Rx 161131,31 paquetes, valor que difiere significativamente del resto de localidades, principalmente de CEBADAS1 puesto que en aquel lugar se Rx 5992,03 paquetes. (tabla 3 ).

Con la arquitectura Atheros localidades CEBADAS2 y CEBADAS3 se Rx 229768,30 y 216276,30 paquetes respectivamente, los mismos que difieren significativamente del resto de tratamientos, principalmente de la arquitectura Atheros CEBADAS1 con el cual se pudo registrar una Rx de 3556,75 paquetes. (tabla 4).

Paquetes E en la Rx, arquitectura Atheros y Broadcom

Al utilizar la arquitectura Atheros no se registraron errores de Rx de paquetes, valores que difieren significativamente de la Broadcom, en la cual se encontró errores de Rx en las localidades CEBADAS 1, 2 y 3, con valores de 3,80, 16,00 y 2,75 paquetes. (tabla 4).

\section{Paquetes Tx, arquitectura Atheros y Broadcom}

De los resultados obtenidos claramente se observó durante el periodo de monitoreo la red WiFi con la arquitectura Atheros se Tx 661980,46 paquetes, valor que difiere significativamente de la arquitectura Broadcom, puesto que con ella se pudo Tx 45033,34 paquetes. (tabla 3). 
Tabla 3. Resultados de comparación entre arquitecturas y localidades en paquetes

\begin{tabular}{|c|c|c|c|c|c|c|c|c|c|c|c|c|c|c|}
\hline \multirow{3}{*}{$\begin{array}{l}\text { Variables/ } \\
\text { Paquetes }\end{array}$} & \multicolumn{4}{|c|}{ Arquitecturas } & \multirow{3}{*}{$\begin{array}{l}\text { Sig } \\
* *\end{array}$} & \multicolumn{8}{|c|}{ Localidades } & \multirow{3}{*}{$\begin{array}{r}\text { Sig } \\
* *\end{array}$} \\
\hline & \multicolumn{2}{|l|}{ Atheros } & \multicolumn{2}{|c|}{ Broadcom } & & \multicolumn{2}{|c|}{ CEBADAS1 } & \multicolumn{2}{|c|}{ CEBADAS2 } & \multicolumn{2}{|c|}{ CEBADAS3 } & \multicolumn{2}{|c|}{ CEBADAS4 } & \\
\hline & 128129,45 & $\mathrm{a}$ & 35490,54 & $\mathrm{~b}$ & & 5992,03 & $\mathrm{c}$ & 161131,31 & $\mathrm{a}$ & 113303,85 & $\mathrm{~b}$ & 35726,77 & $\mathrm{c}$ & \\
\hline Error & 0,00 & $\mathrm{~b}$ & 5,64 & $\mathrm{a}$ & $* *$ & 1,95 & $\mathrm{~b}$ & 8,21 & $\mathrm{a}$ & 1,41 & $\mathrm{bc}$ & 0,00 & $\mathrm{c}$ & $* *$ \\
\hline Tx & 661980,46 & a & 45033,34 & $\mathrm{~b}$ & $* *$ & 8971,87 & $\mathrm{~b}$ & 587398,69 & $\mathrm{a}$ & 629080,69 & a & 124502,54 & $\mathrm{~b}$ & $* *$ \\
\hline Error & 0,00 & $\mathrm{~b}$ & 518,51 & $\mathrm{a}$ & $* *$ & 8,21 & $\mathrm{~b}$ & 1044,51 & $\mathrm{a}$ & 10,77 & $\mathrm{~b}$ & 0,13 & $b$ & $* *$ \\
\hline
\end{tabular}

Tabla 4. Resultados de comparación entre arquitecturas en paquetes

\begin{tabular}{|c|c|c|c|c|c|c|c|c|c|}
\hline \multirow{3}{*}{$\begin{array}{c}\begin{array}{c}\text { Variables/ } \\
\text { Paquetes }\end{array} \\
\mathrm{Rx}\end{array}$} & \multicolumn{9}{|c|}{ Atheros } \\
\hline & \multicolumn{2}{|c|}{ CEBADAS1 } & \multicolumn{2}{|l|}{ CEBADAS2 } & \multicolumn{2}{|l|}{ CEBADAS3 } & \multicolumn{2}{|c|}{ CEBADAS4 } & \multirow{2}{*}{$\frac{\text { Sig }}{* *}$} \\
\hline & 3556,75 & $\mathrm{~d}$ & 229768,30 & $\mathrm{a}$ & 216276,30 & $\mathrm{a}$ & 62916,45 & $\mathrm{bc}$ & \\
\hline Error & 0,00 & $\mathrm{c}$ & 0,00 & $\mathrm{c}$ & 0,00 & $\mathrm{c}$ & 0,00 & $\mathrm{c}$ & $* *$ \\
\hline $\mathrm{Tx}$ & 6530,25 & $\mathrm{~b}$ & 1094569,35 & $\mathrm{a}$ & 1308270,55 & $\mathrm{a}$ & 238551,70 & $\mathrm{~b}$ & $* *$ \\
\hline \multirow[t]{2}{*}{ Error } & 0,00 & $\mathrm{~b}$ & 0,00 & $\mathrm{~b}$ & 0,00 & $\mathrm{~b}$ & 0,00 & $\mathrm{~b}$ & $* *$ \\
\hline & \multicolumn{9}{|c|}{ Broadcom } \\
\hline $\mathrm{Rx}$ & 8134,00 & $\mathrm{~cd}$ & 102961,25 & $\mathrm{~b}$ & 22215,65 & $\mathrm{~cd}$ & 8651,25 & $\mathrm{~cd}$ & $* *$ \\
\hline Error & 3,80 & $\mathrm{~b}$ & 16,00 & $\mathrm{a}$ & 2,75 & $\mathrm{~b}$ & 0,00 & $\mathrm{c}$ & $* *$ \\
\hline $\mathrm{Tx}$ & 10984,05 & $\mathrm{~b}$ & 135471,45 & $\mathrm{~b}$ & 25123,85 & $\mathrm{~b}$ & 8554,00 & $\mathrm{~b}$ & $* *$ \\
\hline Error & 16,00 & $\mathrm{~b}$ & 2036,80 & $\mathrm{a}$ & 21,00 & $\mathrm{~b}$ & 0,25 & $\mathrm{~b}$ & $* *$ \\
\hline
\end{tabular}

En la localidad CEBADAS3 se Tx 629080,69 paquetes, valor que difiere significativamente del resto de localidades, principalmente de CEBADAS1 puesto que en aquel lugar se Tx 8971,87 paquetes. (tabla 3).

Con la arquitectura Atheros localidad CEBADAS3 y CEBADAS2 se registró 1308270,55 y 1094569,35 paquetes de Tx respectivamente, los mismos que difieren significativamente del resto de tratamientos, principalmente de la arquitectura Atheros CEBADAS1 en la cual se registró una Tx de 6530,25 paquetes. (tabla 4). 
Paquetes E en la Tx, arquitecturas Atheros y Broadcom

Al utilizar la arquitectura Atheros no se registraron errores de Tx de paquetes, valores que difieren significativamente de la arquitectura Broadcom, en la cual se encontró errores de Rx en las localidades de CEBADAS 1, 2, 3 y 4, con valores de 16,00, 2036,80, 21,00 y 0,25 paquetes respectivamente. (tabla 4). Sin embargo estos valores no podemos contrastar con ninguna otra investigación, porque no existen publicaciones.

A los datos encontrados en la investigación pueden ser determinados con lo que refiere (Teknlife, 2017), Qualcomm desarrolla una gama de plataformas tribanda, que combinan las soluciones de Qualcomm Atheros y las tecnologías WiFi y WiGig, y están pensadas para mejorar el rendimiento de los dispositivos móviles en redes sin cables. También (National Instruments, 2013), indica que Qualcomm Atheros mejora la velocidad y cobertura de las pruebas de WLAN usando el transceptor vectorial de señales PXI.

Tabla 5. Resultados de comparación entre arquitecturas y localidades en Mbytes

\begin{tabular}{|c|c|c|c|c|c|c|c|c|c|c|c|c|c|c|}
\hline \multirow{3}{*}{$\begin{array}{c}\begin{array}{c}\text { Variables/ } \\
\text { Mbytes }\end{array} \\
\mathrm{Rx} \\
\end{array}$} & \multicolumn{4}{|c|}{ Arquitecturas } & \multirow{3}{*}{$\begin{array}{c}\text { Sig } \\
\mathrm{ns} \\
\end{array}$} & \multicolumn{8}{|c|}{ Localidades } & \multirow{3}{*}{$\begin{array}{r}\text { Sig } \\
* *\end{array}$} \\
\hline & \multicolumn{2}{|c|}{ Atheros } & \multicolumn{2}{|c|}{ Broadcom } & & \multicolumn{2}{|c|}{ CEBADAS1 } & \multicolumn{2}{|c|}{ CEBADAS2 } & \multicolumn{2}{|c|}{ CEBADAS3 } & \multicolumn{2}{|c|}{ CEBADAS4 } & \\
\hline & 35,70 & $\mathrm{a}$ & 34,66 & $\mathrm{a}$ & & 5,93 & c & 82,68 & $\mathrm{a}$ & 39,62 & $\mathrm{~b}$ & 10,34 & $\mathrm{c}$ & \\
\hline $\mathrm{Tx}$ & 131,06 & $a$ & 43,98 & $b$ & $* *$ & 7,18 & $b$ & 167,88 & $\mathrm{a}$ & 141,59 & $\mathrm{a}$ & 21,36 & b & $* *$ \\
\hline
\end{tabular}

Tabla 6. Resultados de comparación entre Arquitecturas en Mbytes

\begin{tabular}{|c|c|c|c|c|c|c|c|c|c|c|c|c|c|c|c|c|c|}
\hline \multirow{3}{*}{$\begin{array}{l}\begin{array}{c}\text { Variables/ } \\
\text { Mbytes }\end{array} \\
\mathrm{Rx}\end{array}$} & \multicolumn{8}{|c|}{ Atheros } & \multicolumn{8}{|c|}{ Broadcom } & \multirow{3}{*}{\begin{tabular}{|l} 
Sig \\
**
\end{tabular}} \\
\hline & \multicolumn{2}{|c|}{ CEBADAS1 } & \multicolumn{2}{|c|}{ CEBADAS2 } & \multicolumn{2}{|c|}{ CEBADAS3 } & \multicolumn{2}{|c|}{ CEBADAS4 } & \multicolumn{2}{|c|}{ CEBADAS1 } & \multicolumn{2}{|c|}{ CEBADAS2 } & \multicolumn{2}{|c|}{ CEBADAS3 } & \multicolumn{2}{|c|}{ CEBADAS4 } & \\
\hline & 3,88 & d & 66,10 & $\mathrm{~b}$ & 60,55 & $\mathrm{~b}$ & 12,25 & $\mathrm{~cd}$ & 7,94 & $\mathrm{~cd}$ & 100,55 & $\mathrm{a}$ & 21,69 & c & 8,45 & $\mathrm{~cd}$ & \\
\hline $\mathrm{Tx}$ & 3,51 & $\mathrm{~d}$ & 212,10 & $\mathrm{c}$ & 274,38 & $\mathrm{a}$ & 34,25 & d & 10,73 & d & 132,30 & b & 24,54 & d & 8,35 & d & $* *$ \\
\hline
\end{tabular}


Mb Rx, arquitectura Atheros y Broadcom

En la localidad CEBADAS2 se registró una Rx de 82,68 Mb de información, valor que difiere significativamente del resto de localidades, principalmente de CEBADAS 1 y 4 puesto que en aquellos lugares se Rx 5,94 y 10,34 Mb de información. (tabla 5).

Con la arquitectura Broadcom localidad CEBADAS2 se Rx 100,55 Mb de información, la cual difiere significativamente del resto de tratamientos, principalmente de Atheros CEBADAS1 con la cual registró una $\mathrm{Rx}$ de 3,88 Mb de información. (tabla 6).

Mb Tx, arquitectura Atheros y Broadcom

Con la arquitectura Atheros se Tx 131,06 Mb de información, valor que difiere significativamente de la arquitectura Broadcom puesto que con ella se pudo Tx 43,98 $\mathrm{Mb}$ de información.

En las localidades CEBADAS2 y 3 se Tx 167,88 y 141,59 Mb de información, valores que difieren significativamente del resto de localidades, principalmente de CEBADAS 1 y 4 en los cuales se Tx 7,18 y 21,36 MB. (tabla 5).

Con la arquitectura Atheros localidad CEBADAS3 se registró una Tx correspondiente a 274,38 Mb de información, el cual difiere significativamente del resto de tratamientos, principalmente del Atheros CEBADAS1 con la cual se registró una Tx de 3,51 Mb de información. Sin embargo estos valores no podemos contrastar con ninguna otra investigación, ya que no existen publicaciones sobre estos parámetros. 
Proceso de Selección, arquitectura más optima

Considerando la Teoría Fisher, que es la relación que existe entre el CMT (Cuadrado Medio de los Tratamientos) sobre el CME (Cuadrado Medio del Error), (Tejedor, F. 1999), tenemos que:

$\mathrm{FC}<\mathrm{FT}(0,05$ y 0,01$) \quad$ ns

FC $>$ FT $(0,05<0,01) \quad *$

FC> FT $(0,01) \quad * *$

Donde $\mathrm{P}<0,01$ y $\mathrm{P}<0,05$ es la relación que existe entre el: Modelo Total, Repeticiones, Arquitecturas, Localidades e Interacciones entre las Arquitecturas y Localidades con el Error, para comprobar si existe significancia. (tabla 7). Según el Análisis de Varianza se determinó que tanto para los Mbytes, paquetes Rx, Tx y sus respectivos errores entre las arquitecturas Atheros y Broadcom, localidades e interacción se encontró diferencias significativas $(\mathrm{P}<0.01)$, por lo tanto la arquitectura más óptima para la implementación de redes Wi-Fi auto extensible en el GAD de Cebadas fue la Arquitectura hardware Atheros.

Tabla 7. Presentación de resultados para la arquitectura más optima

\begin{tabular}{|c|c|c|c|c|c|c|c|c|}
\hline \multirow{2}{*}{$\begin{array}{l}\text { Fuente de } \\
\text { Variación }\end{array}$} & \multicolumn{4}{|c|}{$\begin{array}{l}\text { Fisher Calculado } \\
\text { (FC) }\end{array}$} & & \multicolumn{2}{|c|}{$\begin{array}{c}\text { Fisher Teórico } \\
\text { (FT) }\end{array}$} & \\
\hline & $\operatorname{Rx}($ Paquetes $)$ & Error & Tx(Paquetes) & Error & & $\mathbf{0 , 0 5}$ & $\mathbf{0 , 0 1}$ & \\
\hline Repeticiones & 0,95 & 0,53 & 0,86 & 0,98 & $<$ & 1,67 & 2,04 & ns \\
\hline Arquitecturas & 91,25 & 221,64 & 152,10 & 12849,78 & $>$ & 3,91 & 6,83 & ** \\
\hline Locales & 58,27 & 87,68 & 45,05 & 12242,66 & $>$ & 2,67 & 3,93 & ** \\
\hline Int. $\mathrm{AB}$ & 19,83 & 87,68 & 36,52 & 12242,66 & $>$ & 2,67 & 3,93 & ** \\
\hline
\end{tabular}




\section{Conclusiones.}

La arquitectura hardware Atheros fue la seleccionada para el diseño de redes Wi-Fi auto extensible en el Gobierno Autónomo Descentralizado de Cebadas, porque existe diferencias altamente significativas en los diferentes tratamientos.

De la comparación entre arquitecturas y localidades, los paquetes en la $\mathrm{Rx}$ y Tx de la arquitectura Broadcom son ampliamente superados por la arquitectura Atheros, por lo tanto existe una diferencia altamente siginificativa $(* *)$ entre arquitecturas.

Con los datos obtenidos en la investigación, la arquitectura hardware Atheros es la más óptima para la implementación de redes Wi-Fi auto extensible, ya que se recepto 128129,45 paquetes, valor que difiere significativamente de la arquitectura Broadcom puesto que con ella se pudo receptar 35490,54 paquetes.

El análisis de la tecnología WDS para el diseño de redes WI-fi auto extensible en las arquitecturas hardware Atheros y Broadcom y su implementación en el Gobierno Autónomo Descentralizado de Cebadas, permitió solucionar la problemática en cuanto a conectividad, movilidad, que existía en el GAD, brindando disponibilidad total para los usuarios.

En el proceso de configuración de los routeres, es muy importante copiar la dirección MAC Address WiFi, ya que esta dirección MAC es distinta a la que trae el Router pegada en la cubierta, que corresponde a la MAC de la tarjeta ethernet, no de la inalámbrica, ya que para realizar la conexion es indispensable ingresar la dirección MAC Address WiFi. 
Para la implantación de redes Wi-Fi o cableadas en las distintas instituciones y organizaciones

de la parroquia es muy importante hacer estudios sobre la seguridad en redes, porque al estar conectados los equipos a la red estos puedes ser vulnerados por personas mal intencionadas.

\section{Referencias.}

Almeida, E. \& Coello, A. (2010). Diseño e implementación de una red inalámbrica bajo servidor linux para la Universidad Técnica de Manabí extensión Bahía de Caráquez.

Bandaancha. (2010). Manual WDS. Recuperado de: http://wiki.bandaancha.st/Manual_WDS.

Bellido, W. (2013). Ethical Hacking: Hacking de Red Inalámbrica Wifi

Broadcom. (2015). Conectando la infraestructura de banda ancha Gigabit. Recuperado de: https://www.broadcom.com/.

Dagnino, J. (2014). Análisis de varianza.

Flores, M. (2010). Estudio de la calidad de servicio en la coexistencia entre nodos WLAN 802.11b, g y 802.11 e.

Hiertz, G., Denteneer, D., Max, S., Taori, R., Cardona, J., Berlemann, L., Walke, B. (2010). “IEEE 802.11s: The WLAN mesh standard,” IEEE Wireless Communications, vol. 17, no. 1, pp. 104111. http://dx.doi.org/10.1109/MWC.2010.5416357.

National Instruments. (2013). Qualcomm Atheros mejora la velocidad y cobertura de las pruebas de WLAN usando el transceptor vectorial de señales PXI y el software LabVIEW de National Instruments. Recuperado de http://sine.ni.com/cs/app/doc/p/id/cs-15189\#

PDOT. (Plan de Desarrollo y Ordenamiento Territorial). 2011. Parroquia Cebadas, Ecuador. pp. 511.

Redeweb. (2014). Spansion, el núcleo de sus diseños. Revista española de electrónica. (718): 19-26.

Rodriguez, R. (2010). Diseño de estudio de ingeniería de comunicación inalámbrica e implementación de un sistema de pruebas para el gobierno municipal de Colta, mediante la aplicación de WDS.

Román L. (2016). Análisis del desempeño en un enlace descendente de redes basadas en los estándares IEEE 802.11b, IEEE 802.11n y WDS.

Tejedor, F. (1999). Análisis de la varianza. Shaum. Madrid: La Muralla S.A.

Teknlife. (2017). Wilocity is now part of Qualcomm Atheros, Inc. 
Tello, L. (2010). Análisis de la tecnología WDS y su aplicación en el diseño de infraestructura de red inalámbrica en ambiente open source caso práctico: Fundación desarrollo solidario.

Tung, L., Shih, W., Cho, T., Sun, Y., Chen, M. (2007). “TCP throughput enhancement over wireless mesh networks," IEEE Communications Magazine, vol. 45, no. 11, pp. 64-70. http://dx.doi.org/10.1109/MCOM.2007.4378323.

Zaggoulos, G. \& Nix, A. (2008). WLAN/WDS performance using directive antennas in highly mobile scenarios: experimental results. 